\title{
Metabolic targeting of hypoxia and HIF-1 alpha in colorectal cancer potentiates the therapeutic efficacy of oxaliplatin
}

\author{
Ching-Chow Chen, Tzu-Tang Wei, Yi-Ting Lin, Shao-Pu Tang, Jiou-Cun Tsai \\ Department and Graduate Institute of Pharmacology, College Medicine, National Taiwan University, Taiwan
}

Background: The stabilization of hypoxia inducible factor-1 alpha (HIF-1 alpha) protein plays an important role in the gene transcription in hypoxia. Overexpression of HIF-1 alpha has been reported in various human cancers. It is degraded under normoxic condition by proline hydroxylation through prolyl hydroxylase domain-containing protein (PHD), leading to Von Hippel-Lindau (VHL) E3-ubiquitin ligase-mediated ubiquitination and rapid degradation by proteasome. Succinate is produced when PHD hydroxylates HIF-1 alpha, implying that elevated succinate is parallel with an increase of PHD activity in normoxia.

Methods: Succinate level in cells and mouse tissues was measured by LC-MS analysis. Migration was measured by matrigel plug assay. In vivo angiogenesis assay was performed by Directed In Vivo Angiogenesis Assay (DIVAA) kit (Trevigen).

Results: We found that DNA methyltransferase (DNMT) inhibitor zebularine could down-regulate HIF-1 alpha expression in various colorectal cancer (CRC) cells. Zebularine enhanced the degradation of HIF-1 alpha protein through inducing its hydroxylation. Metabolomic analysis showed that zebularine reversed the reduced succinate level in hypoxia, indicating an increase in PHD activity. Zebularine also reduced angiogenesis and potentiated the anti-cancer effect of oxaliplatin in colorectal cancer (CRC) cells and in azoxymethane (AOM)/dextran sulfate sodium (DSS)-induced CRC mouse model.

Conclusion: This finding provides a new pharmacological strategy of increase in HIF-1 alpha hydroxylation to overcome oxaliplatin resistance and enhance anti-CRC therapy. 